\title{
Tighter Approximation Bounds for Minimum CDS in Wireless Ad Hoc Networks`
}

\author{
Minming $\mathrm{Li}^{1}$, Peng-Jun Wan ${ }^{2}$, and Frances $\mathrm{Yao}^{1}$ \\ 1 Department of Computer Science, City University of Hong Kong \\ minmli@cs.cityu.edu.hk, csfyao@cityu.edu.hk \\ 2 Department of Computer Science, Illinois Institute of Technology \\ wan@cs.iit.edu
}

\begin{abstract}
Connected dominating set (CDS) has a wide range of applications in wireless ad hoc networks. A number of approximation algorithms for constructing a small CDS in wireless ad hoc networks have been proposed in the literature. The majority of these algorithms follow a general two-phased approach. The first phase constructs a dominating set, and the second phase selects additional nodes to interconnect the nodes in the dominating set. In the performance analyses of these two-phased algorithms, the relation between the independence number $\alpha$ and the connected domination number $\gamma_{c}$ of a unit-disk graph plays the key role. The best-known relation between them is $\alpha \leq 3 \frac{2}{3} \gamma_{c}+1$. In this paper, we prove that $\alpha \leq 3.4306 \gamma_{c}+4.8185$. This relation leads to tighter upper bounds on the approximation ratios of two approximation algorithms proposed in the literature.
\end{abstract}

\section{Introduction}

Connected dominating set (CDS) has a wide range of applications in wireless ad hoc networks (cf. a recent survey [3] and references therein). Consider a wireless ad hoc network with undirected communication topology $G=(V, E)$. A CDS of $G$ is a subset $U \subset V$ satisfying that each node in $V \backslash U$ is adjacent to at least one node in $U$ and the subgraph of $G$ induced by $U$ is connected. A number of distributed algorithms for constructing a small CDS in wireless ad hoc networks have been proposed in the literature. The majority of these distributed algorithms follow a general two-phased approach 12448101112. The first phase constructs a dominating set, and the nodes in the dominating set are called dominators. The second phase selects additional nodes, called connectors, which together with the dominators induce a connected topology. The algorithms in 112481011 differ in how to select the dominators and connectors. For example, the algorithm in 2] selects the dominators using the Chvatal's greedy

\footnotetext{
* This work was partially supported by Research Grants Council of Hong Kong SAR under Project No. CityU 122807 and No. CityU 117408, and National Basic Research Program of China Grant 2007CB807900 and 2007CB807901. Peng-Jun Wan was supported in part by National Science Foundation of USA under grants CNS-0831831 and CNS-0916666.
} 
algorithm [5] for Set Cover, the algorithms in [110] select an arbitrary maximal independent set (MIS) as the dominating set, and all the algorithms in [481112] choose a special MIS with 2-hop separation property as the dominating set.

The approximation ratios of these two-phased algorithms 12481011 have been analyzed when the communication topology is a unit-disk graph (UDG). For a wireless ad hoc network in which all nodes lie in a plane and have equal maximum transmission radii normalized to one, its communication topology $G=(V, E)$ is often modelled by a UDG in which there is an edge between two nodes if and only if their Euclidean distance is at most one. Except the algorithms in 210 which have logarithmic and linear approximations ratios respectively, all other algorithms in 14481112 have constant approximation ratios. The algorithm in [1] targets at distributed construction of CDS in linear time and linear messages. With this objective, it trades the size of the CDS with the time complexity, and thus its approximation ratio is a large constant (but less than 192). The analyses of the algorithms in 481112 rely on the relation between the independence number (the size of a maximum independent set) $\alpha$ and the connected domination number (the size of a minimum connected dominating set) $\gamma_{c}$ of a connected UDG $G$. A loose relation $\alpha \leq 4 \gamma_{c}+1$ was obtained in [1], which implies an upper bound of 8 on the approximation ratios of both algorithms in 411. A refined relation $\alpha \leq 3.8 \gamma_{c}+1.2$ was discovered in [13]. With such a refined relation, the upper bound on the approximation ratios of both algorithms in 411] was reduced from 8 to 7.6, and an upper bound of $5.8+\ln 5 \approx 7.41$ on the approximation ratio of the algorithms in [8] was derived (the bound $4.8+\ln 5 \approx 6.41$ in 8 ] was incorrect). The best-known relation $\alpha \leq 3 \frac{2}{3} \gamma_{c}+1$ if $G$ has at least two nodes was recently proven in [12. As a result, the upper bound on the approximation ratio of the algorithm in [11 was further reduced to $7 \frac{1}{3}$ in [12]. Another greedy approximation algorithm was also proposed in 12 and its approximation ratio was proven to be bounded by $6 \frac{7}{18}$.

In this paper, we first prove a further improved relation $\alpha \leq 3.4306 \gamma_{c}+4.8185$ in Section 3. The proof for this bound employs an integrated area and length argument, and involves some other interesting extreme geometric problems which are studied in Section 2. Subsequently in Section 4, we provide tighter analyses of the approximation algorithm in [11] and the other greedy algorithm in [12]. We prove that the approximation ratio of the former algorithm is at most 6.862 and the approximation ratio of the latter algorithm is at most 6.075 .

We remark that a recent paper [7] claimed that for any connected UDG $G$,

$$
\alpha \leq 3.453 \gamma_{c}+8.291
$$

However, as discovered in 12, the proof for a key geometric extreme property underlying such claim was missing, and such proof is far from being apparent or easy. Such property is rigorously proved in Lemma 5. Consequently, the bound claimed in 7 can be treated at most as a conjecture at the time of its publication rather than a proven result.

In the remaining of this section, we introduce some terms and notations. For any point $u$ and any $r>0$, we use $\operatorname{disk}_{r}(u)$ to denote the closed disk of radius $r$ centered at $u$, and circle $_{r}(u)$ to denote the boundary circle of disk $k_{r}(u)$. A path 
or a polygon is said to be inscribed in a circle if all its vertices lie on the circle. The Lebesgue measure (or area) of a measurable set $A \subset \mathbb{R}^{2}$ is denoted by $|A|$. The topological boundary of a set $A \subset \mathbb{R}^{2}$ is denoted by $\partial A$. For the simplicity of presentation, the line segment between two points $u$ and $v$ and its length are both denoted by $u v$ by slightly abusing the notation, but the actual meaning can be clearly told from the context.

\section{Canonical Polygons and Inscribed Polygons}

Suppose that $s, o$ and $t$ are three points from the left to the right on a horizontal line with $o s=1$ and $o t=0.5$. For any pair of points $u$ and $v$ on $\operatorname{circle}_{1 / \sqrt{3}}(o)$, let $\widehat{u v}$ be the arc in circle $_{1 / \sqrt{3}}(o)$ from $u$ to $v$ in the counterclockwise manner. Denote by $\phi$ the radian of $\widehat{u v}$, and let $k=\lceil\phi /(\pi / 3)\rceil$. We construct a path $Q$ of $k$ edges from $u$ to $v$ with all vertices on $\widehat{u v}$ as follows: If $\phi$ is an integer multiple of $\frac{\pi}{3}$, then all edges of $Q$ are tangent to circle $_{0.5}(o)$; otherwise, all edges except the $\lceil k / 2\rceil$-th edge are tangent to circle $_{1 / \sqrt{3}}(o)$ (we remark that in this case, the $\lceil k / 2\rceil$-th edge is disjoint from circle $\left._{1 / \sqrt{3}}(o)\right)$. The path $Q$ is referred to as the canonical path inscribed in circle $e_{1 / \sqrt{3}}(o)$ from $u$ to $v$.

For any point $u$ which lies on the right side the the vertical line through $t$, we construct a polygon $P$ as follows: let $u_{1}$ and $u_{2}$ be the two points on circle $_{1 / \sqrt{3}}(o)$ such that the two line segments $u_{1} u$ and $u_{2} u$ are tangent to circle $_{1 / \sqrt{3}}(o)$ and $u_{1}$ is above the line st. Then, $P$ is surrounded by $u_{1} u, u_{2} u$ and the canonical path from $u_{1}$ to $u_{2}$. The polygon $P$ is referred to as the canonical polygon of $u$. The point $u$ is called the base vertex of $P$, and the angle $\theta=\arccos \frac{1}{2 o u}$ is called the base angle of $P$. Note that if $u$ is on the ray ot, then $P$ is symmetric with respect to the line ot, and the area of $P \cap \operatorname{disk}_{1.5}(s)$ is a function of the base angle $\theta$, which is denoted by $f(\theta)$. In this section, we will derive the explicit expression of $f(\theta)$ and explore some useful properties of the function $f(\theta)$. We will also prove that for any canonical polygon $P,\left|P \cap \operatorname{disk}_{1.5}(s)\right| \geq f(\theta)$ where $\theta$ is the base angle of $P$.

We first introduce a geometric function $g$ on $[0, \pi]$ defined as follows. For any $\theta \in[0, \pi]$, let $v$ be a point on circle $_{1 / 2}(o)$ satisfying that $\angle$ tov $=\theta$ and $v$ is above st. Let $w$ be the point on $\operatorname{circle}_{1.5}(s)$ satisfying that $v w$ is tangent to circle $_{1 / 2}(o)$ and $w$ lies to the right of $v$. Then, $g(\theta)$ is defined to be the area of the region surrounded by arc $t w$ and the three line segments ot, ov and $v w$ (see Figure 1). The next lemma presents the explicit expressions of $g(\theta)$ and its first and second order derivatives.

Due to space limit, we omit some of the proofs in this version.

Lemma 1. Let $\beta=\theta-\arccos \frac{1+2 \cos \theta}{3}$. Then,

$$
\begin{aligned}
g(\theta) & =\frac{9}{8} \beta-\frac{3}{4} \sin \beta+\frac{\sqrt{6}}{4} \sin \frac{\beta}{2}, \\
g^{\prime}(\theta) & =\frac{13}{8}-1.5 \cos \beta,
\end{aligned}
$$




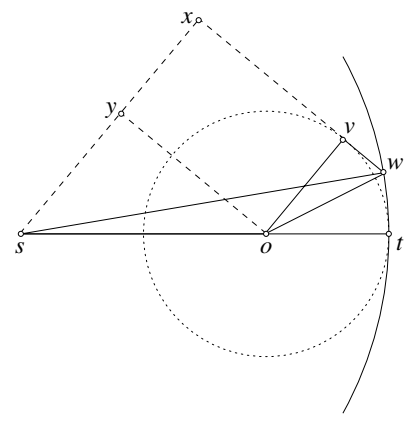

Fig. 1. Calucaltion of $\angle w s t$ and $g(\theta)$

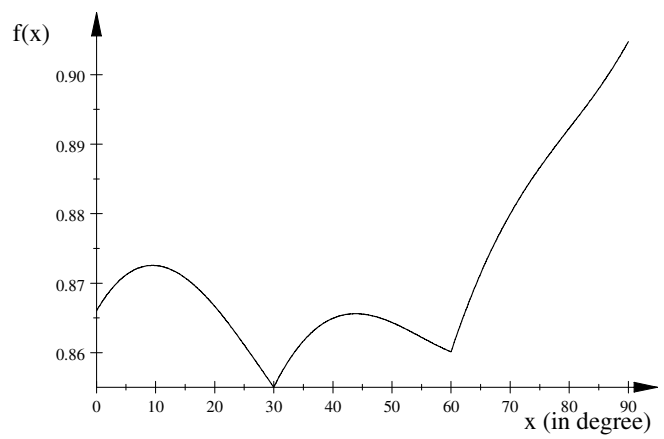

Fig. 2. The curve of $f$ on $\left[0^{\circ}, 90^{\circ}\right)$

$$
g^{\prime \prime}(\theta)=1.5\left(1-\sqrt{1-\frac{1}{2+\cos \theta}}\right) \sin \beta .
$$

In addition, $g$ is increasing and convex on $[0, \pi]$, while both $g^{\prime}$ and $g^{\prime \prime}$ are increasing on $\left[0, \frac{\pi}{2}\right]$.

It is easy to show that $f(\theta)=2 g(\theta)+h(\theta)$, where

$$
h(\theta)=\frac{1}{4 \sqrt{3}}\left\lfloor 6-\frac{\theta}{\frac{\pi}{6}}\right\rfloor+\frac{1}{6} \sin 2\left(\left(6-\left\lfloor 6-\frac{\theta}{\frac{\pi}{6}}\right\rfloor\right) \frac{\pi}{6}-\theta\right) .
$$

Figure 2 is the curve of $f$ on $\left[0^{\circ}, 90^{\circ}\right)$. We observe and will prove later that $f$ is increasing on $\left[60^{\circ}, 90^{\circ}\right)$. However, on either of the two intervals $\left[0^{\circ}, 30^{\circ}\right]$ and $\left[30^{\circ}, 60^{\circ}\right], f$ is neither monotone, nor concave, and nor convex. Fortunately, on either of these two intervals $f$ has the following weak but still nice quasi-concave property: $f$ is said to be quasi-concave on an interval $[a, b] \subset\left[0^{\circ}, 90^{\circ}\right)$ if for each triple of increasing values $\theta_{1}, \theta_{2}, \theta_{3}$ in $[a, b], f\left(\theta_{2}\right) \geq \min \left\{f\left(\theta_{1}\right), f\left(\theta_{3}\right)\right\}$.

Lemma 2. $f$ is quasi-concave on $\left[0, \frac{\pi}{6}\right]$ and $\left[\frac{\pi}{6}, \frac{\pi}{3}\right]$ respectively, and increasing on $\left[\frac{\pi}{3}, \frac{\pi}{2}\right)$. 
Denote $f\left(\frac{\pi}{6}\right)$ by $\sigma$. Then,

$$
\sigma=\frac{\sqrt{3}}{6}-\frac{1}{2}+\frac{\sqrt{8+2 \sqrt{3}}}{4}+\frac{3 \pi}{8}-\frac{9}{4} \arccos \frac{1+\sqrt{3}}{3} \approx 0.85505328 .
$$

It is easy to verify that $f(0)=\sqrt{3} / 2$ and $f\left(32^{\circ}\right)<f\left(\frac{\pi}{3}\right)<f\left(34^{\circ}\right)$. So, by Lemma 2 we have the following corollary.

Corollary 1. The minimum of $f$ on the interval $\left[0^{\circ}, 90^{\circ}\right)$ (respectively, $\left[32^{\circ}, 90^{\circ}\right)$ and $\left[34^{\circ}, 90^{\circ}\right)$ ) is achieved at $30^{\circ}$ (respectively, $32^{\circ}$ and $60^{\circ}$ ).

Finally, we prove the following extreme property of the canonical polygons.

Lemma 3. For any canonical polygon $P$ with base angle $\theta,\left|P \cap \operatorname{disk}_{1.5}(s)\right| \geq$ $f(\theta)$.

Next, we prove the following lemma about inscribed polygons.

Lemma 4. Suppose that $P$ is a polygon inscribed in circle $e_{1 / \sqrt{3}}(o)$ satisfying that $\operatorname{disk}_{0.5}(o) \subseteq P$. Then,

$$
\begin{aligned}
& |P|>\sqrt{3} / 2, \\
& \left|P \cap \operatorname{disk}_{1.5}(s)\right| \geq \sigma .
\end{aligned}
$$

\section{Independence Number vs. Connected Domination Number}

In this section, we present an improved upper bound on the independence number in terms of the connected domination number.

Theorem 1. Let $\alpha$ and $\gamma_{c}$ be the independence number and connected domination number of a connected UDG G. Then,

$$
\alpha \leq 3.4306 \gamma_{c}+4.8185 \text {. }
$$

We prove the above theorem by an integrated area and length argument. Let $U$ be a minimum CDS of $G$, and define

$$
\Omega=\bigcup_{u \in U} \operatorname{disk}_{1.5}(u) .
$$

Consider a maximum independent set $I$ of $G$. We construct the Voronoi diagram defined by $I$. For each $o \in I$, we use $\operatorname{Vor}(o)$ to denote its Voronoi cell and call the set $\operatorname{Vor}(o) \cap \Omega$ as the truncated Voronoi cell of $o$. Clearly, $|\Omega|$ is the total area of truncated Voronoi cells of all nodes in $I$. We partition $I$ into two subsets $I_{1}$ and $I_{2}$ defined by

$$
\begin{aligned}
& I_{1}=\left\{o \in I: \operatorname{disk}_{1 / \sqrt{3}}(o) \subset \Omega\right\}, \\
& I_{2}=I \backslash I_{1} .
\end{aligned}
$$

Denote by $\alpha_{1}$ and $\alpha_{2}$ the size of $I_{1}$ and $I_{2}$ respectively. The next lemma provides a lower bound on each truncated Voronoi cell. 


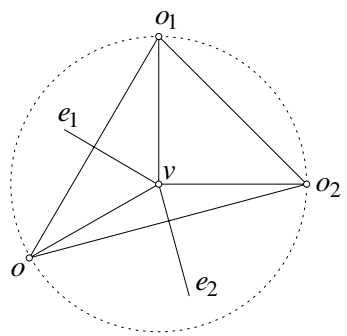

Fig. 3. Any vertex of $\operatorname{Vor}(o)$ is apart from $o$ by at least $1 / \sqrt{3}$

Lemma 5. For each o in $I_{1}$ (respectively, $I_{2}$ ), the area of its truncated Voronoi cell is at least $\sqrt{3} / 2$ (respectively, $\sigma$ ).

Proof. Since the pairwise distances of the points in $I$ are at least one, the distance between $o$ and each side of $\operatorname{Vor}(o)$ is at least 0.5 and consequently $\operatorname{disk}_{0.5}(o) \subset$ $\operatorname{Vor}(o)$. Next, we show that no vertex of $\operatorname{Vor}(o)$ is inside $\operatorname{dis}_{1 / \sqrt{3}}(o)$. Let $v$ be a vertex of $\operatorname{Vor}(o)$, and $e_{1}$ and $e_{2}$ be the two sides of $\operatorname{Vor}(o)$ incident to $v$ (see figure 3). Let $o_{1}$ (respectively, $o_{2}$ ) be the point which is symmetric to $o$ with respect to $e_{1}$ (respectively, $e_{2}$ ). Then, both $o_{1}$ and $o_{2}$ belong to $I$, and hence the three sides of $\triangle \mathrm{OO}_{1} \mathrm{O}_{2}$ are all at least 2. Clearly, $v$ is the center of $\triangle \mathrm{oO}_{1} \mathrm{O}_{2}$. Since at least one of the three central angles of $\triangle \mathrm{OO}_{1} \mathrm{O}_{2}$ is at most $120^{\circ}$, the circumscribing radius of $\triangle o_{1} O_{2}$ is at least $1 / \sqrt{3}$. Thus, ov $\geq 1 / \sqrt{3}$.

Let $s$ be the node in the MCDS $U$ closest to $o$. Then, $o \in \operatorname{disk}_{1}(s)$. If $\operatorname{disk}_{1 / \sqrt{3}}(o) \subseteq \operatorname{Vor}(o)$, then $|\operatorname{Vor}(o) \cap \Omega| \geq\left|\operatorname{disk}_{1 / \sqrt{3}}(o) \cap \operatorname{disk}_{1.5}(s)\right|>\sqrt{3} / 2$. So, we assume $d i s k_{1 / \sqrt{3}}(o)$ is not fully contained in $\operatorname{Vor}(o)$. Then $\operatorname{Vor}(o)$ intersects circle $_{1 / \sqrt{3}}(o)$. We construct a polygon $P \subseteq \operatorname{Vor}(o)$ satisfying that $P$ is inscribed in circle $_{1 / \sqrt{3}}(o)$ and $\operatorname{disk}_{0.5}(o) \subseteq P \subseteq \operatorname{Vor}(o)$. Let $Q$ be the sequence of intersecting points between $\operatorname{Vor}(o)$ and circle $_{1 / \sqrt{3}}(o)$ in the counterclockwise order. For each pair of successive $u$ and $v$ in $Q$, if $\angle u o v \leq \frac{\pi}{3}$, we add to $P$ a side between $u$ and $v$; otherwise, we add to $P$ a path inscribed in the arc from $u$ to $v$ satisfying that each edge in this path is either tangent to or disjoint from circle $_{1 / \sqrt{3}}(o)$ (see Figure 4). The resulting polygon $P$ meets the requirement. By Lemma 4, $|P| \geq \sqrt{3} / 2$.

If $o \in I_{1}$, then

$$
P \subseteq \operatorname{Vor}(o) \cap \operatorname{disk}_{1 / \sqrt{3}}(o) \subseteq \operatorname{Vor}(o) \cap \Omega,
$$

hence

$$
|\operatorname{Vor}(o) \cap \Omega| \geq|P| \geq \sqrt{3} / 2 \text {. }
$$

Now, we assume that $o \in I_{2}$. Note that $\left|P \cap \operatorname{disk}_{1.5}(s)\right|$ grows when moving $o$ away from $s$ along a fixed radius of $\operatorname{disk}_{1.5}(s)$. By Lemma $4\left|P \cap \operatorname{disk}_{1.5}(s)\right| \geq \sigma$. Since

$$
P \cap \operatorname{disk}_{1.5}(s) \subseteq \operatorname{Vor}(o) \cap \Omega,
$$




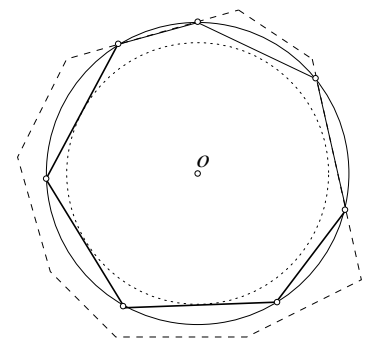

Fig. 4. Inserting operations

we have

$$
|\operatorname{Vor}(o) \cap \Omega| \geq\left|P \cap \operatorname{disk}_{1.5}(s)\right| \geq \sigma .
$$

We define

$$
\Omega^{\prime}=\bigcup_{v \in U} d i s k_{1.5-1 / \sqrt{3}}(v) .
$$

The next lemma gives an upper bound on the length of $\partial \Omega^{\prime}$.

Lemma 6. The length of $\partial \Omega^{\prime}$ is at most $2(1-1 / \sqrt{3}) \alpha_{2}$.

By Lemma 5 .

$$
|\Omega| \geq \frac{\sqrt{3}}{2} \alpha_{1}+\sigma \alpha_{2}=\frac{\sqrt{3}}{2} \alpha-\left(\frac{\sqrt{3}}{2}-\sigma\right) \alpha_{2},
$$

which implies

$$
\alpha \leq \frac{|\Omega|}{\frac{\sqrt{3}}{2}}+\left(1-\frac{\sigma}{\frac{\sqrt{3}}{2}}\right) \alpha_{2} .
$$

It is easy to prove by induction on $\gamma_{c}$ that

$$
|\Omega| \leq \frac{9}{2}\left(\left(\gamma_{c}-1\right)\left(\arcsin \frac{1}{3}+\frac{\sqrt{8}}{9}\right)+\frac{\pi}{2}\right),
$$

and the length of of $\partial \Omega^{\prime}$ is at most

$$
2\left(3-\frac{2}{\sqrt{3}}\right)\left(\left(\gamma_{c}-1\right) \arcsin \frac{1}{3-\frac{2}{\sqrt{3}}}+\frac{\pi}{2}\right) .
$$

By Lemma 6.

$$
\begin{aligned}
\alpha_{2} \leq & \frac{2\left(3-\frac{2}{\sqrt{3}}\right)\left(\left(\gamma_{c}-1\right) \arcsin \frac{1}{3-\frac{2}{\sqrt{3}}}+\frac{\pi}{2}\right)}{2\left(1-\frac{1}{\sqrt{3}}\right)} \\
& =\frac{\sqrt{3}+7}{2}\left(\left(\gamma_{c}-1\right) \arcsin \frac{1}{3-\frac{2}{\sqrt{3}}}+\frac{\pi}{2}\right) .
\end{aligned}
$$


The three inequalities (11), (2) and (3) imply altogether that $\alpha$ is at most

$$
\begin{aligned}
& \left(\gamma_{c}-1\right)\left(\begin{array}{c}
\sqrt{27}\left(\arcsin \frac{1}{3}+\frac{\sqrt{8}}{9}\right) \\
+\frac{\left(1-\frac{\sigma}{\frac{\sqrt{3}}{2}}\right)(\sqrt{3}+7)}{2} \arcsin \frac{1}{3-\frac{2}{\sqrt{3}}}
\end{array}\right)+\frac{\pi}{2}\left(\sqrt{27}+\left(1-\frac{\sigma}{\frac{\sqrt{3}}{2}}\right) \frac{\sqrt{3}+7}{2}\right) \\
& \approx 3.4305176 \gamma_{c}+4.8184688 .
\end{aligned}
$$

Thus, Theorem 1 follows.

\section{Tighter Approximation Ratios}

In this section, we derive tighter bounds on the approximation ratio of the distributed algorithm proposed in [1] and the other greedy algorithm proposed in 12. For the convenience of presentation, we call them WAF and WWY respectively. Let $G=(V, E)$ be a unit-disk graph. We denote by $\alpha$ and $\gamma_{c}$ the independence number and connected domination number of $G$ respectively. For any finite set $S$, we use $|S|$ to denote the cardinality of $S$.

The CDS produced by the algorithm WAF consists of a maximal independent set $I$ and a set $C$ of connectors. Specifically, let $T$ be an arbitrary rooted spanning tree of $G$. The set $I$ is selected in the first-fit manner in the breadth-first-search ordering in $T$. Let $s$ be the neighbor of the root of $T$ which is adjacent to the largest number of nodes in $I$. Then, $C$ consists of $s$ and the parents (in $T$ ) of the nodes in $I \backslash I(s)$. It was proved in [11] that $I \cup C$ is a CDS and $|I \cup C| \leq 8 \gamma_{c}-1$. Later on, two progressively improved tighter bounds $7.6 \gamma_{c}+1.4$ and $7 \frac{1}{3} \gamma_{c}$ were obtained in [13] and [12] respectively. The next theorem further improves the bound on $|I \cup C|$.

Theorem 2. The CDS produced by the algorithm $\boldsymbol{W A \boldsymbol { F }}$ has size at most 6 . $862 \gamma_{c}+8.637$.

Proof. Let $I$ and $C$ be the set of nodes selected by the algorithm WAF in the first phase and the second phase respectively. Since $|C| \leq|I|-1$, we have

$$
|I \cup C| \leq 2|I|-1 \leq 2\left(3.4306 \gamma_{c}+4.8185\right)-1 \leq 6.862 \gamma_{c}+8.637 .
$$

So, the theorem follows.

In the next, we study the algorithm WWY. The first phase of this algorithm is the same as the algorithm WAF, and we let $I$ be the selected maximal independent set. But the second phase selects the connectors in a more economic way. For any subset $U \subseteq V \backslash I$, let $q(U)$ be the number of connected components in $G[I \cup U]$. For any $U \subseteq V \backslash I$ and any $w \in V \backslash I$, we define

$$
\triangle_{w} q(U)=q(U)-q(U \cup\{x\}) .
$$

The value $\triangle_{w} q(U)$ is referred to as the gain of $w$ with respect to $U$. The following lemma was proved in 12 . 
Lemma 7. Suppose that $q(U)>1$ for some $U \subseteq V \backslash I$. Then, there exists a $w \in V \backslash(I \cup U)$ such that $\triangle_{w} q(U) \geq \max \left\{1,\left\lceil q(U) / \gamma_{c}\right\rceil-1\right\}$.

The second phase of the algorithm WWY runs as follows. We use $C$ to denote the sequence of selected connectors. Initially $C$ is empty. While $q(C)>1$, choose a node $w \in V \backslash(I \cup C)$ with maximum gain with respect to $C$ and add $w$ to $C$. When $q(C)=1$, then $I \cup C$ is a CDS. It was proved in 12 that $|I \cup C| \leq 6 \frac{7}{18} \gamma_{c}$. We derive a tighter bound on the output CDS in the theorem below.

Theorem 3. The CDS produced by the algorithm $\boldsymbol{W} \boldsymbol{W} \boldsymbol{Y}$ has size at most 6 . $075 \gamma_{c}+5.425$.

Proof. Let $I$ and $C$ be the set of nodes selected by the algorithm WWY in the first phase and the second phase respectively. If $\gamma_{c}=1$, then $|I| \leq 5$ and $|C| \leq 1$, hence $|I \cup C| \leq 6$. Thus, the theorem holds trivially if $\gamma_{c}=1$. If $|I| \leq 3 \gamma_{c}+2$, then $|I \cup C| \leq 2|I|-1 \leq 6 \gamma_{c}+3$, and the theorem also holds. From now on, we assume that $\gamma_{c} \geq 2$ and $|I|>3 \gamma_{c}+2$.

We break $C$ into three contiguous (and possibly empty) subsequences $C_{1}, C_{2}$ and $C_{3}$ as follows. $C_{1}$ is the shortest prefix of $C$ satisfying that $q\left(C_{1}\right) \leq 3 \gamma_{c}+2$, and $C_{1} \cup C_{2}$ is the shortest prefix of $C$ satisfying that $q\left(C_{1} \cup C_{2}\right) \leq 2 \gamma_{c}+1$. We can prove that

$$
\begin{aligned}
& \left|C_{1}\right| \leq\left\{\begin{array}{c}
\frac{|I|}{3}-\gamma_{c} \text { if } q\left(C_{1}\right) \leq 3 \gamma_{c}+1, \\
\frac{|I|-2}{3}-\gamma_{c} \text { if } q\left(C_{1}\right)=3 \gamma_{c}+2 ;
\end{array}\right. \\
& \left|C_{2}\right| \leq\left\{\begin{array}{c}
\frac{\gamma_{c}}{2} \text { if } q\left(C_{1}\right) \leq 3 \gamma_{c}+1 \\
\frac{\gamma_{c}+1}{2} \text { if } q\left(C_{1}\right)=3 \gamma_{c}+2 ;
\end{array}\right. \\
& \left|C_{3}\right| \leq 2 \gamma_{c}-1 .
\end{aligned}
$$

From the first two inequalities, we have

$$
\left|C_{1} \cup C_{2}\right| \leq \frac{|I|}{3}-\frac{\gamma_{c}}{2} .
$$

Using the third inequality, we have

$$
|C| \leq \frac{|I|}{3}-\frac{\gamma_{c}}{2}+2 \gamma_{c}-1=\frac{|I|}{3}+\frac{3}{2} \gamma_{c}-1 .
$$

So,

$$
\begin{aligned}
& |I \cup C| \leq \frac{4|I|}{3}+\frac{3}{2} \gamma_{c}-1 \\
\leq & \frac{4}{3}\left(3.4306 \gamma_{c}+4.8185\right)+\frac{3}{2} \gamma_{c}-1 \\
\leq & 6.075 \gamma_{c}+5.425 .
\end{aligned}
$$

Thus, the theorem follows. 


\section{Discussions}

In this paper, we obtained a tighter relation between the independence number and connected domination number of a connected UDG. We actually proved the following stronger result on packing. Let $V$ be a set of $n$ nodes of a connected dominating set, and $\Gamma$ be the unions of unit-disks centered at $V$. Then, we can pack in $\Gamma$ at most $3.4306 n+4.8185$ points whose pairwise distances are greater than or equal to one. We'd like to emphasize that here we allow two points packed in $\Gamma$ to have distance equal to one. On the other hand, a packing of $3 n+3$ points in $\Gamma$ whose pairwise distances are greater than one was presented in 12 . It was also conjectured $3 n+3$ is the exact bound. Thus, there is still a gap between the bound $3.4306 n+4.8185$ derived in this paper and the conjectured bound $3 n+3$.

\section{References}

1. Alzoubi, K.M., Wan, P.-J., Frieder, O.: Message-Optimal Connected Dominating Sets in Mobile Ad Hoc Networks. In: ACM Mobihoc (2002)

2. Bharghavan, V., Das, B.: Routing in Ad Hoc Networks Using Minimum Connected Dominating Sets. In: IEEE ICC (1997)

3. Blum, J., Ding, M., Cheng, X.: Applications of Connected Dominating Sets in Wireless Networks. In: Du, D.-Z., Pardalos, P. (eds.) Handbook of Combinatorial Optimization, pp. 329-369. Kluwer Academic Publisher, Dordrecht (2004)

4. Cadei, M., Cheng, X., Du, D.-Z.: Connected Domination in Ad Hoc Wireless Networks. In: Proc. 6th International Conference on Computer Science and Informatics (2002)

5. Chvátal, V.: A greedy heuristic for the set-covering problem. Mathematics of Operation Research 4(3), 233-235 (1979)

6. Clark, B.N., Colbourn, C.J., Johnson, D.S.: Unit Disk Graphs. Discrete Mathematics 86, 165-177 (1990)

7. Funke, S., Kesselman, A., Meyer, U., Segal, M.: A simple improved distributed algorithm for minimum CDS in unit disk graphs. ACM Transactions on Sensor Networks 2(3), 444-453 (2006)

8. Li, Y.S., Thai, M.T., Wang, F., Yi, C.-W., Wan, P.-J., Du, D.-Z.: On Greedy Construction of Connected Dominating Sets in Wireless Networks. Journal on Wireless Communications and Mobile Computing 5(8), 927-932 (2005)

9. Lin, F., Yang, X. (eds.): Geometric Measure Theory: An Introduction. International Press (2003)

10. Stojmenovic, I., Seddigh, M., Zunic, J.: Dominating sets and neighbor elimination based broadcasting algorithms in wireless networks. In: Proc. IEEE Hawaii Int. Conf. on System Sciences (January 2001)

11. Wan, P.-J., Alzoubi, K.M., Frieder, O.: Distributed Construction of Connected Dominating Set in Wireless Ad Hoc Network. ACM/Springer Mobile Networks and Applications 9(2), 141-149 (2004); A preliminary version of this paper appeared in IEEE INFOCOM (2002) 
12. Wan, P.-J., Wang, L., Yao, F.: Two-Phased Approximation Algorithms for Minimum CDS in Wireless Ad Hoc Networks. In: IEEE ICDCS 2008, pp. 337-344 (2008)

13. Wu, W., Du, H., Jia, X., Li, Y., Huang, S.C.-H.: Minimum connected dominating sets and maximal independent sets in unit disk graphs. Theoretical Computer Science 352(1-3), 1-7 (2006) 